\title{
Lo que pueden decir de la comida chilena los diccionarios fundacionales. Primera parte
}

\author{
What foundational diccionaries can say about Chilean food. \\ Part One
}

\author{
Soledad Chávez Fajardo \\ schavez@uchile.cl \\ Universidad de Chile \\ Academia de la Lengua
}

Resumen: La finalidad de este artículo es presentar una investigación en curso: dar cuenta de lo que hay detrás de un artículo lexicográfico relacionado con el campo semántico de los alimentos en la lexicografía hispanoamericana fundacional. La investigación tiene dos objetivos: presentar una propuesta de tipologización de esta información y dar cuenta de las funciones que poseen estos artículos lexicográficos. Estas funciones serán dos, que tienen que ver con el papel normativo que tenían estos diccionarios y, por otro lado, con el papel etnográfico, respecto a mostrar, sea directa o indirectamente, una realidad hispanoamericana.

Palabras clave: comida, Chile, diccionarios, sigle XIX, lexicografía.

Abstract: The purpose of this article is to present an ongoing investigation: to give an account of what is behind a lexicographical article related to the semantic field of food in the foundational Hispanic-American lexicography. The research has two objectives: to present a proposal for typologizing this information and to give an account of the functions that these lexicographical articles have. These functions will be two, which have to do with the normative role that these dictionaries had and, on the other hand, with the ethnographic role, with respect to showing, directly or indirectly, a Latin American reality.

Keywords: food, Chile, dictionaries, 19th. century, lexicographie.

\section{Acerca de la función de los primeros diccionarios monolingües publicados en Hispanoamérica}

Desde una óptica de condiciones de producción, la aparición de diccionarios monolingües en Chile y en Hispanoamérica en general, a partir del siglo XIX, ha sido producto de los procesos estandarizadores. Estos procesos fueron, a su vez, el resultado de la conformación de los nuevos Estados nación. Dentro del proceso estandarizador, sobre todo en su praxis didáctica, el modelo que ha primado en Hispanoamérica en general y sobre todo en Chile fue el racionalista (Geeraerts 2003), heredero de la escuela que Andrés Bello llevó a cabo en Chile. Es decir, prevaleció el modelo que normaba la lengua española siguiendo una lengua ejemplar, es decir, el estándar o pauta de referencia para las variedades. Esta lengua ejemplar se entendía como una variedad no pluricéntrica, sino monocéntrica y el referente para trabajar en ella era la Real Academia Española y cada una de sus publicaciones. 


\section{Soledad Chávez Fajardo. Lo que pueden decir de la comida chilena los diccionarios fundacionales. Primera parte}

Es por esta razón que estos diccionarios, por lo general, se ceñían a una ejemplaridad y su función era, las más veces, dar cuenta de las incorrecciones, barbarismos, disparates, vulgarismos, entre otras calificaciones. Sin embargo, al revisar y leer estos diccionarios en detalle se puede detectar que en un artículo lexicográfico existe una suerte de tensión, entre normar y describir, entre validar y censurar una palabra diferencial o determinado uso. En efecto, si se revisan los lemarios de estos diccionarios, se constata que suelen abundar palabras características de estas variedades (voces diferenciales, sea en signo o en significado), así como de aspectos relacionados con la normatividad (por ejemplo, cómo se escribe una palabra, cómo se debe adaptar un extranjerismo, cómo se conjuga un verbo, por qué no puede aceptarse determinada polisemia o sinonimia, diferencial, entre tantos casos). Justamente este es el principal contenido de gran parte de estos diccionarios: lo diferencial sea para darlo a conocer y para que el diccionario académico reconozca estas voces; sea para censurar la diatopía porque existen sinónimos ya asentados dentro de la lengua ejemplar. A su vez, lo normativo para que el usuario pueda conocer y usar la lengua ejemplar de la mejor manera posible.

En esta dinámica es que entra en juego el campo semántico de los alimentos. En efecto, las palabras de este campo abundan en los diccionarios, sea para dar cuenta de un referente que no ha sido lematizado en el diccionario académico, sea porque hay un uso diferencial que, a ojos vistas del lexicógrafo hispanoamericano, es innecesario o puede dar el autor alguna propuesta de hispanización o censura de algún extranjerismo, entre otras. Justamente el propósito de esta investigación en curso es dar cuenta de lo que hay detrás de un artículo lexicográfico relacionado con el campo semántico de los alimentos y cómo se puede tipologizar el tratamiento que estos primeros lexicógrafos hispanoamericanos hicieron de estas palabras.

No hay que olvidar que estos diccionarios, desde una óptica discursiva y pragmática, se entienden como discursos que contienen actos de respuesta. En efecto, un usuario consulta y la información que aporta un artículo lexicográfico responde. Sin embargo ¿qué responde? ¿es adecuada esta respuesta? ¿es válida, del momento que se trabaja con una lengua ejemplar? Anteriormente había comentado (Chávez Fajardo 2015) que si se aplicara, dentro de las lógicas de la acción comunicativa habermasiana, ${ }^{1}$ el principio de rectitud, este se desbarataría ante un acto de respuesta como el de estos primeros diccionarios. En rigor, estos artículos lexicográficos se pueden entender como actos perlocucionarios, porque buscan generar una modificación en el comportamiento del hablante. En ellos, por ejemplo, abundan los verbos ilocutivos: se advierte, se ordena, se objeta, se aprueba, se censura. La finalidad, empero, no se queda dentro de los espacios de la ilocución: se busca generar una respuesta en el receptor. ${ }^{2}$ Esta respuesta va desde la modificación de un artículo lexicográfico

\footnotetext{
1 Dialéctica que no es para nada mía, puesto que Luis Fernando Lara en su Teoría del diccionario monolingüe (1997), trabajó por primera vez con estos postulados dentro del ámbito de la lexicografía. En sus reflexiones releyó la teoría de la acción comunicativa de Bühler y sobre todo la de Habermas para conformar su teoría de la lexicografía española, ni más ni menos.

2 Un ensayo que ayuda a entender cómo es la dinámica desde un punto de vista pragmático es el de Darío Rojas 2010 «Estandarización lingüística y pragmática del diccionario: “forma y función” de los diccionarios de provincialismos chilenos».
} 
Soledad Chávez Fajardo. Lo que pueden decir de la comida chilena los diccionarios fundacionales. Primera parte

en el diccionario académico hasta el conocimiento de cierta norma lingüística. Justamente el campo semántico de los alimentos entra en esta dinámica, como se mostrará más adelante, con una relevante tipología de actos perlocucionarios e ilocucionarios.

\section{Leer y estudiar un diccionario: leer diccionarios}

Para este estudio he trabajado con un diccionario-base para mi búsqueda, que es el Diccionario de chilenismos y de otras voces y locuciones viciosas, del sacerdote diocesano Manuel Antonio Román. Román publicó este diccionario entre 1901 y 1918 en cinco volúmenes. Es considerado el diccionario hispanoamericano de la lexicografía fundacional más extenso. Contiene más de quince mil entradas y los volúmenes en total suman casi tres mil páginas. A su vez, hay muchísima lematización interna en el diccionario, por lo que las palabras tratadas y definidas, en rigor, exceden el número del lemario oficial. He decidido trabajar con este diccionario como base, justamente, por su extensión y por la profusión de detalles e información que el sacerdote trata del campo léxico que nos convoca: el de los alimentos.

Sin embargo, creo que es insuficiente un estudio lexicológico que tenga como base un diccionario si no se lo lee a partir de un diálogo constante con otros diccionarios que forman parte de su contexto y de su tradición. En efecto, un diccionario no debe leerse solo, sino más bien en relación con otros diccionarios y obras afines, es decir, como interdiscursos (Pêcheux 2005 [1975]), en tanto entramados de formaciones discursivas, donde se establecen relaciones de alianza o de contradicción entre ellos. Justamente, en estos artículos lexicográficos se pueden ver repeticiones, reformulaciones o refutaciones de información existente, en este caso, de palabras relacionadas con el campo semántico de los alimentos. Para ello se ha utilizado un paradigma más o menos fijo, distintivo de ciertas tradiciones lexicográficas; por ejemplo, los diccionarios de lengua española general más representativos, diccionarios etimológicos, diccionarios normativos o diccionarios enciclopédicos, entre otros. De esta forma, el artículo lexicográfico dedicado al campo de los alimentos de Román será una suerte de patrón en donde se podrá determinar, en rigor, si los discursos contemporáneos al del sacerdote daban cuenta de la misma información. Por lo tanto, si bien el rastreo de palabras del campo de los alimentos fue a partir de una obra, a lo largo del análisis fue imprescindible consultar y dialogar con otras obras lexicográficas y afines. Es por esta misma razón que he utilizado, además, una obra paralela, sobre todo considerando el alto número de indigenismos: el Diccionario etimológico de las voces chilenas derivadas de las lenguas indígenas americanas de Rodolfo Lenz (1979 [1904-1910]). Lenz, alemán radicado en Chile antes de cumplir treinta años, fue uno de los fundadores del Departamento de Lingüística de la Universidad de Chile. Ajeno a estos procesos codificadores racionalistas y alejado de esta relación entre la lengua española y la RAE (para esto, ver Chávez Fajardo 2011), su trabajo tuvo un carácter más bien descriptivo y etnográfico. Si bien en su diccionario se pueden encontrar bastantes imprecisiones y erratas (algo usual en la lexicografía en general, dicho sea de paso), es este un trabajo aún no superado dentro de la lexicografía española y etimológica. 


\section{Soledad Chávez Fajardo. Lo que pueden decir de la comida chilena los diccionarios fundacionales. Primera parte}

\section{Metodología y propuesta de análisis}

Es esta una investigación en curso y la finalidad es aportar en un campo específico en los estudios de lexicología histórica del español de América, en parte y del español de Chile, sobre todo. He detectado que un número relevante de artículos lexicográficos relacionados con el campo semántico de la alimentación forman parte de un acervo cultural olvidado o restringido a un espacio reducido de hablantes, o bien, a un léxico pasivo en la actualidad. A su vez, hay poca información respecto a la historia de su normativización, por ejemplo, o cómo se fueron asentando y presentando algunas de estas palabras. Una tarea futura será la de recabar esta información y disponerla a manera de un lemario de carácter lexicológico y lexicográfico histórico. Esto lo señalo como un objetivo a largo plazo, puesto que el objetivo de esta investigación es presentar una tipologización de una muestra de estos artículos lexicográficos para mostrar cómo la primera lexicografía hispanoamericana trataba las palabras relacionadas con este campo semántico. En otras palabras, deseo estudiar la función de los artículos lexicográficos de dicho campo en la lexicografía fundacional chilena.

Para el estudio he seleccionado las tres primeras letras del Diccionario; serían, siguiendo la dinámica de Román, las cuatro primeras letras, porque el sacerdote, muy de sus tiempos, consideró el dígrafo <ch> como letra independiente. Esta selección arroja el 26,6\% del Diccionario, lo que es una muestra representativa mas no idónea, porque solo considera el primer y parte del segundo volumen. Es decir, he trabajado solo con el Román lexicógrafo en el rango de 1901 a 1911, dejando de lado al segundo Román, el que va de 1913 a 1918. Sin embargo, esta falencia se subsana por el carácter de «en curso» de estos primeros resultados tentativos.

\begin{tabular}{|c|c|c|}
\hline Letra & Número de entradas & Porcentaje \\
\hline A & 1.224 & $7,89 \%$ \\
\hline$B$ & 582 & $3,75 \%$ \\
\hline C & 1.858 & $11,97 \%$ \\
\hline $\mathrm{Ch}$ & 380 & $2,45 \%$ \\
\hline$D$ & 772 & $4,97 \%$ \\
\hline E & 1.020 & $6,57 \%$ \\
\hline$F$ & 367 & $2,36 \%$ \\
\hline G & 399 & $2,57 \%$ \\
\hline $\mathrm{H}$ & 323 & $2,08 \%$ \\
\hline $\mathrm{I}$ & 447 & $2,88 \%$ \\
\hline $\mathrm{J}$ & 125 & $0,81 \%$ \\
\hline $\mathrm{K}$ & 14 & $0,09 \%$ \\
\hline $\mathrm{L}$ & 455 & $2,93 \%$ \\
\hline $\mathrm{Ll}$ & 72 & $0,46 \%$ \\
\hline $\mathrm{M}$ & 948 & $6,11 \%$ \\
\hline $\mathrm{N}$ & 200 & $1,29 \%$ \\
\hline$\tilde{\mathrm{N}}$ & 78 & $0,50 \%$ \\
\hline $\mathrm{O}$ & 249 & $1,60 \%$ \\
\hline $\mathrm{P}$ & 1.965 & $12,66 \%$ \\
\hline $\mathrm{Q}$ & 202 & $1,30 \%$ \\
\hline $\mathrm{R}$ & 999 & $6,44 \%$ \\
\hline$S$ & 937 & $6,04 \%$ \\
\hline $\mathrm{T}$ & 1.107 & $7,13 \%$ \\
\hline
\end{tabular}

\begin{tabular}{|l||r|r|}
\hline $\mathrm{U}$ & 123 & $0,79 \%$ \\
\hline \hline $\mathrm{V}$ & 452 & $2,91 \%$ \\
\hline \hline $\mathrm{W}$ & 21 & $0,14 \%$ \\
\hline $\mathrm{X}$ & 3 & $0,02 \%$ \\
\hline \hline $\mathrm{Y}$ & $\mathbf{6 5}$ & $0,42 \%$ \\
\hline \hline $\mathrm{Z}$ & 136 & $0,88 \%$ \\
\hline \hline & $\mathbf{1 5 . 5 2 3}$ & $\mathbf{1 0 0} \%$ \\
\hline \hline
\end{tabular}

Lemario del Diccionario de Román, considerando números de entradas y equivalente en porcentaje. 


\section{Soledad Chávez Fajardo. Lo que pueden decir de la comida chilena los diccionarios fundacionales. Primera parte}

Trabajé con un total de 81 palabras recabadas entre las letras a y c, incluyendo a la $<\mathrm{ch}>$, que son las referentes al campo semántico de la alimentación. Con ellas, según la información que está presente en la microestructura, sobre todo en la definición e información específica, como explicaciones, sinonimias, ejemplificación y citas, he ido tipologizando las funciones de dichos artículos de la siguiente manera:

\begin{tabular}{|c|c|c|}
\hline I & El Diccionario de Román y la RAE & $\begin{array}{l}\text { 1. Dar cuenta de que en el DRAE aparece lematizada la palabra en } \\
\text { cuestión } \\
\text { 2. Dar cuenta de que en el DRAE no aparece el sentido chileno, el } \\
\text { que es presentado en el Diccionario de Román } \\
\text { 3. Pedir a la RAE que lematice la palabra en cuestión } \\
\text { 4. Dar cuenta de que en el DRAE la palabra se escribe de tal o cual } \\
\text { manera, entendida como la forma correcta } \\
\text { 5. Opiniones de Román respecto a las adaptaciones que la RAE hace } \\
\text { de los extranjerismos } \\
\text { 6. Actitud crítica o irónica con la RAE } \\
\text { 7. Pedir que la RAE enmiende la definición o parte de la definición }\end{array}$ \\
\hline III & $\begin{array}{l}\text { Aspectos de normatividad y } \\
\text { corrección }\end{array}$ & $\begin{array}{l}\text { 1. Incorrecciones para Román } \\
\text { 2. Cuestiones de adaptación de palabras de procedencia no } \\
\text { hispánica } \\
\text { 3. Cuestiones de normatividad }\end{array}$ \\
\hline III & $\begin{array}{l}\text { Dar cuenta del referente: "en Chile } \\
\text { existe este alimento" }\end{array}$ & $\begin{array}{l}\text { 1. Alimentos dulces: a) golosinas; b) postres } \\
\text { 2. Alimentos semilíquidos de consistencia espesa } \\
\text { 3. Azúcar: a) derivados a partir de la remolacha } \\
\text { 4. Bebidas alcohólicas } \\
\text { 5. Carnes: a) casquería; b) tipos de carnes } \\
\text { 6. Cereales: a) métodos de cocción de cereales } \\
\text { 7. Comidas frías } \\
\text { 8. Condimentos } \\
\text { 9. Frutas: a) frutas; b) preparaciones con frutas } \\
\text { 10. Guisos } \\
\text { 11. Harinas: a) fritos de masa; b) masas horneadas; c) tipos de } \\
\text { harinas } \\
\text { 12. Hongos } \\
\text { 13. Huevos: a) preparaciones de huevos } \\
\text { 14. Maíz: a) presentaciones del maíz; b) preparaciones con maíz } \\
\text { 15. Pan: a) preparaciones con pan; b) tipos de panes } \\
\text { 16. Papas: a) tipos de papas; b) preparaciones con papas } \\
\text { 17. Quesos: a) tipos de quesos } \\
\text { 18. Tipos de cocción }\end{array}$ \\
\hline
\end{tabular}


Soledad Chávez Fajardo. Lo que pueden decir de la comida chilena los diccionarios fundacionales. Primera parte

\begin{tabular}{|c|c|c|}
\hline & & $\begin{array}{l}\text { 19. Utensilios } \\
\text { 20. Verduras }\end{array}$ \\
\hline IV & Referentes de otras zonas & $\begin{array}{l}\text { 1. Respecto a la cocina mapuche } \\
\text { 2. Respecto a referentes no lematizados o tardíamente lematizados } \\
\text { en el DRAE } \\
\text { 3. Respecto a referentes de otros países hispanoamericanos } \\
\text { 4. Respecto a América toda }\end{array}$ \\
\hline $\mathrm{V}$ & Referentes de ámbitos específicos & a) Mundo de la marina \\
\hline VI & Cuestiones semánticas & $\begin{array}{l}\text { 1. Sinonimia } \\
\text { 2. Imposibilidad de sinonimia } \\
\text { 3. Transiciones semánticas } \\
\text { 4. Remisiones } \\
\end{array}$ \\
\hline VII & Palabras de origen no hispánico & $\begin{array}{l}\text { 1. Anglicismos } \\
\text { 2. Galicismos } \\
\text { 3. Indigenismos }\end{array}$ \\
\hline VIII & $\begin{array}{l}\text { Informaciones etimológicas y de } \\
\text { procedencia }\end{array}$ & $\begin{array}{l}\text { 1. Historia de un alimento y su preparación } \\
\text { 2. Propuestas etimológicas }\end{array}$ \\
\hline
\end{tabular}

En la medida en que la investigación avance aparecerán más matices y tipos en esta propuesta de clasificación. Por ejemplo, en el caso del campo 3 (dar cuenta del referente: «en Chile existe este alimento»), fui detectando subcampos, que son los indicados con letras, los cuales no serán ni por lo mucho los campos y subcampos definitivos. O en el campo 5 , hasta la $<\mathrm{c}>$ solo he encontrado un ámbito específico (la marina), algo que podrá ampliarse más adelante. Lo mismo con las palabras de origen no hispánico, las que pueden extenderse sin duda alguna, a otras tradiciones lingüísticas Siguiendo el orden de los campos y su secuencia, he recabado las siguientes palabras (los subcampos los he reemplazado por números correlativos):

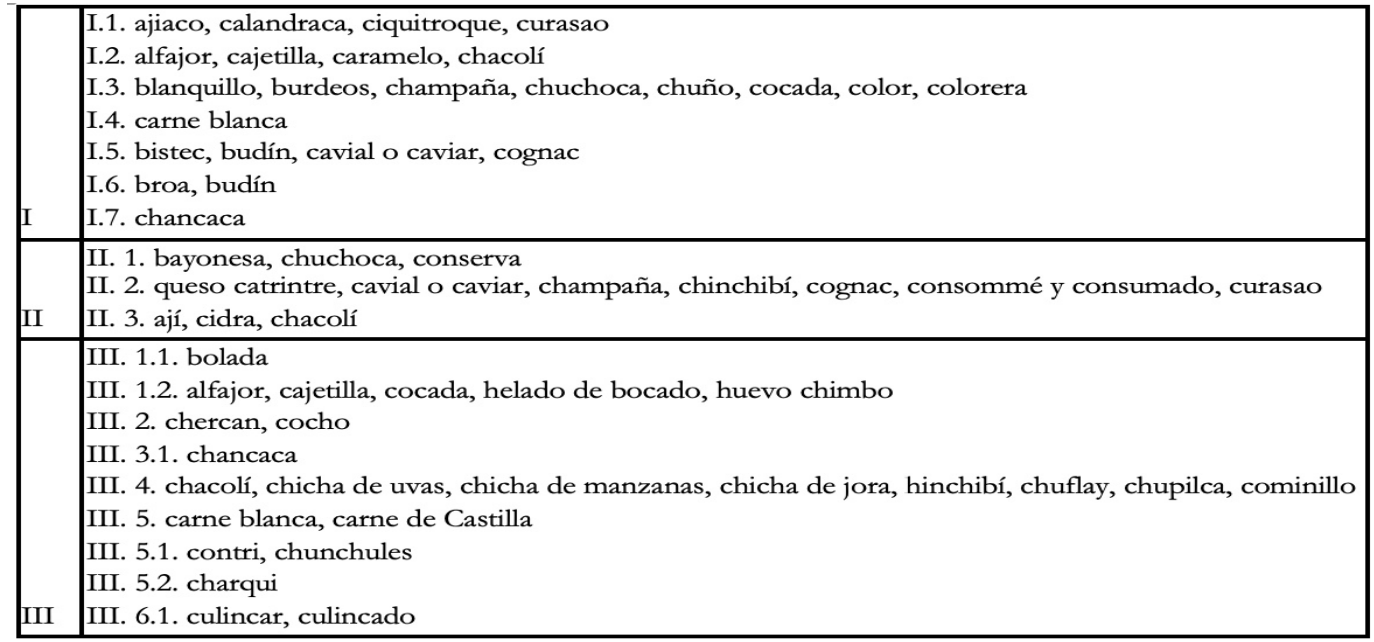




\section{Soledad Chávez Fajardo. Lo que pueden decir de la comida chilena los diccionarios fundacionales. Primera parte}

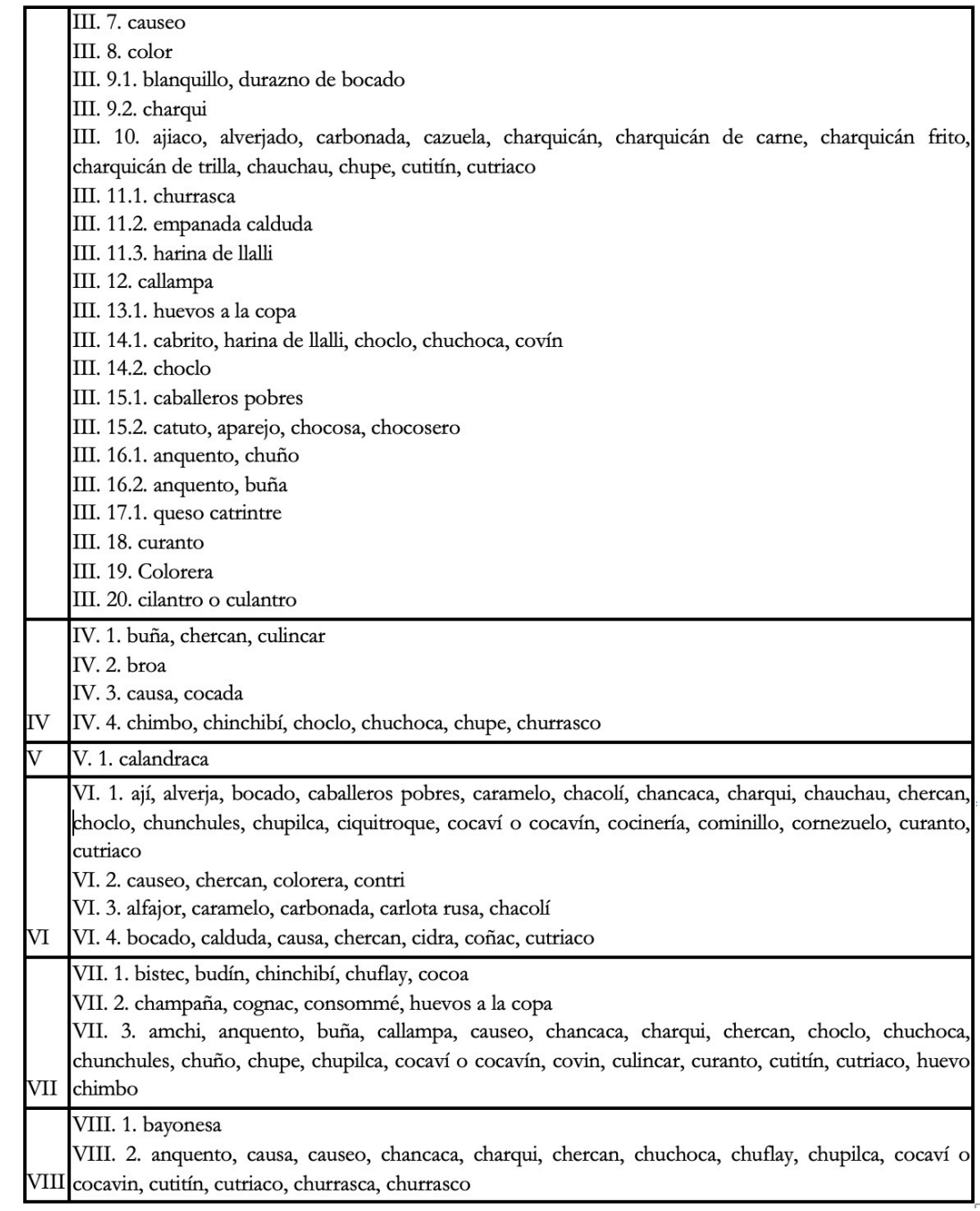

\section{Análisis de cada uno de los campos detectados}

Desarrollaré un análisis de los artículos lexicográficos seleccionados, todos en directa relación con el campo y función recabada. Por cuestiones de espacio en este ensayo me detendré en los dos primeros campos detectados y parte del tercero, quedando para una segunda parte de la presentación los campos restantes. Creo que esto no menoscaba la presente investigación, por ser un trabajo en curso, en primer lugar y porque el análisis es representativo del tipo de análisis que estoy haciendo. Para los cotejos he trabajado sobre todo con las herramientas que tiene la RAE, idóneas para tal efecto: el CORDE, el NTLLE, el Fichero del NDHLE y el recientemente 
Soledad Chávez Fajardo. Lo que pueden decir de la comida chilena los diccionarios fundacionales. Primera parte

aparecido Tesoro de los diccionarios históricos de la lengua española (TDHLE). También he recurrido al Léxico hispanoamericano de Peter Boyd Bowman y al CORDIAM. También para verificar la vigencia, he consultado el Diccionario de americanismos de la ASALE. A su vez, he consultado algunos diccionarios hispanoamericanos, sobre todo si confirmo que no han aparecido referidas en las herramientas mencionadas. Por ejemplo, tal como hacía mención anteriormente, a la par con Román, el Diccionario etimológico de las voces chilenas derivadas de las lenguas indígenas americanas de Rodolfo Lenz (1979 [1904-1910]) será una herramienta de consulta fundamental, sobre todo para los indigenismos o posibles indigenismos, que son bastantes. Porque la cuestión etimológica no entraba totalmente en lo tratado en estas páginas, prácticamente no he usado Corominas, pero está citado en un caso. Seá clave cuando trabaje con algunas propuestas etimológicas, porque Román solía desarrollar bastante este tipo de información en sus artículos lexicográficos, por lo mismo, además, el trabajo constante y en paralelo con Lenz en estos casos es fundamental. Como una segunda etapa de la investigación deberé consultar, además, repertorios gastronómicos chilenos, los que me ayudarán a dilucidar ciertos aspectos que no se resuelvan con las herramientas lingüísticas mencionadas, como el Manual del cocinero práctico chileno (1900), entre otros.

\subsection{El Diccionario de Román y la RAE}

\subsubsection{Dar cuenta de que el en DRAE aparece lematizada la palabra en cuestión}

La noticia de que la palabra aparece en el Diccionario académico es usual en la primera lexicografía hispanoamericana. Es una suerte de visado de que el americanismo «es válido» $\mathrm{y}$ «puede usarse» sin problema.

Si bien ajiaco ya venía siendo incorporado dentro de la tradición española (cfr. en el NTLLE Salvá, Castro y Rossi, editorial Gaspar y Roig, Domínguez), siempre la definición estaba referida al ajiaco en Colombia o Cuba. La definición más general y pensada para toda Hispanoamérica «aunque con significado un poco distinto del que aquí le damos» (Román 1901-1908) es la del Suplemento a la $12^{a}$ edición de la Academia (1884).

En otros casos, como en calandraca, no se hace referencia a Hispanoamérica, si no a la noticia de una palabra lematizada por el Diccionario académico («término de Marina admitido por primera vez, dice Román 1901-1908).

Lo mismo con curasao (con esta grafía en el Diccionario académico en 1899), que tendrá más presencia con la grafía con $\langle\mathrm{z}>$, como en Domínguez, editorial Gaspar y Roig, Zerolo y Gaspar y Gómez (cfr. en el NTLLE). Con $<$ ç $>$ lo pedirá Román y no habrá registros lexicográficos al respecto (sí literarios, como se puede ver en los dos casos que tiene el Fichero).

En el caso de ciquitroque, la preparación que ha sido referida por primera vez por Terreros (cfr. NTLLE), el que equiparaba al pisto. Román nota: «ha sido admitida por primera vez esta voz, pero sin etimología» (1901-1908), que Corominas tampoco logra dilucidar y emparenta con almodrote y su variedad almodroque «viene acaso ciquitroque», propone (1980). 
Soledad Chávez Fajardo. Lo que pueden decir de la comida chilena los diccionarios fundacionales. Primera parte

\subsubsection{Dar cuenta de que en el DRAE no aparece el sentido chileno, el que es presentado en el Diccionario de Román}

Otras informaciones tienen que ver con la información que pueda tener el diccionario académico en torno a un lema. Muchas veces, la definición o explicación será insuficiente o no dará cuenta cabal del sentido chileno.

Por ejemplo, el alfajor característico muchos países hispanoamericanos, entendido como el dulce compuesto por «dos rodajas de masa adheridas una a otra con dulce y a veces recubierta de chocolate, merengue, etc» (DLE), empieza a ser descrito de dicha manera en Chile (Fidelis del Solar 1875, Ortúzar 1893, Román 1901-1908, Medina 1928, cfr. TDHLE) y en Argentina (Granada 1899, Garzón 1910, Segovia 1911, cfr. TDHLE). Hasta la edición usual de 1925, reconocida por ser la primera vez en que la RAE dio cabida a un número importante de palabras procedentes de Hispanoamérica, la definición que había para alfajor era: «pasta hecha de harina de yuca, papelón, piña y jengibre, que se usa en América». Román comentaba, al respecto: «Esta definición, tomada literalmente del Dicc. de Zerolo, no cuadra, por cierto, al alfajor chileno, que es un dulce pequeño, formado de dos piezas de masa más o menos fina y adheridas la una a la otra con manjar blanco o melcocha» (1901-1908).

Para el caso de cajetilla, dulce que prácticamente ha desaparecido del mundo culinario chileno y del que solo Román lematizó, argumenta: «para lo cual no vemos inconveniente, pues la voz cajetilla es también diminutiva de caja, y aunque el Dicc. le dé solamente la acep. de paquete de cigarrillos de papel) (1901-1908).

En el caso del chacoli, por ejemplo, Román argumentaba: «SS. vinariegos de Sud-América y otras regiones, a vosotros os incumbe probar que también conocéis y sabéis fabricar el chacolí. Para nosotros, es el vino hecho con la uva común del país, llamada de viña. En algunas provincias lo confunden con la chicha, porque fermenta sin el orujo; en otras lo llaman vino nuevo» (1908-1911), justamente, porque el sema «Chile» no estuvo especificado hasta la décimo octava edición de 1956 en el DRAE.

\subsubsection{Pedir a la RAE que lematice la palabra en cuestión}

Otra dinámica, que es usual en esta lexicografía fundacional hispanoamericana es pedir que la RAE lematice las palabras en cuestión. Por ejemplo, respecto a referentes americanos es usual esta petición, sobre todo los que dan cuenta de alimentos propiamente americanos. Es recurrente en estos casos que Román cierre los artículos lexicográficos con frases del tipo: «Merece admitirse en el Dicc.»; «En esta forma debe pues entrar en el Dicc»; «Con más razón puede admitirse a chuño, que se usa en Chile, Argentina, Perú y Bolivia, por lo menos» (para chuño); «Con el uso de Colombia y de Méjico, a quienes representan estos dos autores, y con el de Chile, del cual damos testimonio nosotros, hay sobrada razón para que la Academia acepte este vocablo» (para cocada); «Por lo demás, y ya que en castellano no puede tener equivalente, pues la color no se conoce en España, creemos que este chilenismo tiene derecho a la admisión» (para color); «Por la misma razón que color, nos parece que debe ser admitido» (para colorera). 
Soledad Chávez Fajardo. Lo que pueden decir de la comida chilena los diccionarios fundacionales. Primera parte

Tal es el caso de lo que Román hace con el pez comestible blanquillo (prolatillus jugularis), incorporado en la décima quinta edición del DRAE (1925).

Con la chuchoca (molienda de maíz tierno, similar a la polenta), ya lematizado por Salvá, Gaspar y Roig, Domínguez (en su suplemento), Zerolo, Toro y Gómez, Alemany, Rodríguez Navas, incorporado en la décima sexta edición del DRAE (1936), pasando previamente por la primera manual de 1927 (cfr. NTLLE). Con el chuño (fécula de patata), ya lematizado por Salvá, Gaspar y Roig, Domínguez (en su suplemento), Zerolo, Toro y Gómez, incorporado en la décimocuarta edición del DRAE de 1914 (cfr. NTLLE). Con cocada (dulce a base de coco que se extendió a España, además), ya lematizado por Salvá, Castro y Rossi, Gaspar y Roig, Domínguez (en su suplemento), Zerolo, referido por Cuervo en sus Apuntaciones, García Icazbalceta para México, Toro y Gómez, Alemany (cfr. TDHLE). Incorporado en la décima quinta edición del DRAE (1925) sin marca diatópica alguna, lo que da cuenta de su extensión a España. Con color y colorera, por ejemplo, incorporada solamente, dentro de la tradición lexicográfica, en Alemany y Santamaría en su Diccionario de Americanismos (cfr. Fichero).

No solamente son palabras relacionadas con referentes americanos. Suelen ser, además, extranjerismos que dan cuenta de productos absolutamente asentados en la realidad hispánica, los que Román pide lematizar.

En el caso de burdeos, la postura es, incluso, crítica: «Ya es tiempo de que la Academia sea más liberal con los nombres de los vinos y licores que se han hecho de uso general en todo el mundo. Si bien es cierto que no puede incluir en su Dicc. todos los particulares de una o más naciones, también es cierto que no puede excluir los que ya son conocidos y designados con un nombre fijo por toda la gente ilustrada» (1901-1908), señala Román. Por lo que clama que se incluya gran parte del paradigma de los alcoholes: «¿por qué no admite también los de licores nacionales y extranjeros que ya gozan de fama universal?» (1901-1908). El vino ya estaba lematizado en Zerolo y terminó por incorporarse en la décima quinta edición del DRAE de 1925 (cfr. NTLLE).

Lo mismo hizo Román con champaña: «Si ya es conocido en todo el mundo y los mismos españoles lo beben, no hay por qué excluirlo del Dicc.» (1908-1911), ya lematizada de dicha manera por Domínguez, Gaspar y Roig, Salvá (en su suplemento), Toro y Gómez, Pagés, Alemany e incorporado en la décima quinta edición del DRAE de 1925 (cfr. NTLLE).

\subsubsection{Dar cuenta de que en el DRAE la palabra se escribe de tal o cual manera, entendida como la forma correcta}

Otra instancia usual en los diccionarios hispanoamericanos funcionales es instalar el DRAE como la herramienta para determinar cómo se debe escribir una palabra y en esto no se escapa el campo semántico que nos convoca, tal es el caso del artículo lexicográfico carne, en relación con carne blanca: «según el Dicc. solo puede usarse en pl., carnes blancas, y son las de las aves en general y la mayor parte de las que no son de montería» (Román 1901-1908), uso vigente (el plural en el diccionario) hasta la vigésima segunda edición (2001). Respecto al uso en singular, este está detectado con este sentido, en el XVIII (cfr. CORDE, con Bernabé Cobo). 
Soledad Chávez Fajardo. Lo que pueden decir de la comida chilena los diccionarios fundacionales. Primera parte

\subsubsection{Opiniones de Román respecto a las adaptaciones que la RAE hace de los extranjerismos}

Una actitud interesantísima, sobre todo para estudios que tengan que ver con ideologías y actitudes lingüísticas es la que se puede encontrar en esta primera lexicografía hispanoamericana en relación con las decisiones que pueda tomar la RAE, por ejemplo, en lo que tenga que ver con la adaptación de extranjerismos. La idea generalizante de la actitud servil de estos primeros lexicógrafos hacia la institución se desbarata en estos casos.

Por ejemplo, en el caso de bistec, Román afirmaba: «Aunque es mucho más fácil y natural para los que hablan castellano pronunciar $s$ que $f$ antes de $t$, ha optado la Academia por esta última letra y suprimió aquella, cuando en inglés, de donde tomó la palabra (beefteak), tiene las dos» (1901-1908). En efecto, la adaptación fue primero como biftec (duodécima edición, 1884), también por Zerolo, Toro y Gómez, Pagés y Alemany (cfr. NTLLE). Román sería el primero, en mi rastreo, en proponer esta variante que será la que terminará por imponerse y ser lematizada por el DRAE en la décima quinta edición (1925), forma que, según el CORDE, ya era escrita por Bretón de los Herreros en los años veinte del XIX.

En el caso de budín, como el dulce preparado con bizcocho o migas de pan, que es la adaptación que Román proponía, daba cuenta de otras variantes: «Algunos proponen la forma pudín o pudingo pero la más generalizada es budín», a lo que Román pedía: «Resuelva la cuestión la Academia» (1901-1908). Forma que también había lematizado Zerolo y que se incorporó en la décima quinta edición del DRAE de 1925. A partir de la vigésima primera edición (1992), se optó por la variante pudín (cfr. NTLLE).

Para el vino burdeos, junto con el reclamo visto anteriormente para que se lematice, Román argumentaba, respecto al campo semántico del vino y su irregular presencia en el diccionario académico: «A la verdad, no deja de ser raro ver en el Dicc. nombres como cécubo y másico, vinos usados en tiempo de los romanos y llamados así por el lugar en que se producían, y no encontrar los nombres de los vinos usados por los mismos españoles» (1901-1908), el que terminó por incorporarse en la décima quinta edición del DRAE de 1925.

El caso del artículo lexicográfico cavial o caviar, Román pide que haya una postura clara respecto a cómo escribirlo: «Así, con v, escribe estos nombres el Dicc. de la Academia; pero la Gramática de la misma solo trae la forma cabial: ¿a cuál de los dos seguir?» (1901-1908). Si uno hace el rastreo de la palabra en la tradición hispánica, ya aparece como caviar hacia 1447 (las Andanz̧as de Pedro Tafur, CORDE). La primera lematización es en Autoridades como cabial, siguiendo a Cervantes y su Quijote y siguió de dicha forma en sucesivas ediciones hasta la undécima edición (1869). También en Salvá, Castro y Rossi, Domínguez, Gaspar y Roig y Zerolo. Destaco en Terreros su lema serial con bastantes variantes: kavia, kaviac, kaviar o kavial. También Terreros lematizó cavial con remisión al lema serial y cavial también empieza a aparecer en la duodécima edición de la Academia de 1884. La primera lematización como caviar es en Domínguez y es la que termina por generalizarse (cfr. NTLLE).

En el caso de cognac, Román pide hispanizarlo como coñac, que ya estaba en Castro y Rossi y que la RAE termina por lematizar en su décimocuarta edición de 1914 (cfr. NTLLE). 


\section{Soledad Chávez Fajardo. Lo que pueden decir de la comida chilena los diccionarios fundacionales. Primera parte}

\subsubsection{Actitud crítica o irónica con la RAE}

Otra instancia interesante es la actitud, propia de esta primera lexicografía, de dar cuenta de opiniones y actitudes que hoy en día serían absolutamente contrarias a las normas y metodologías lexicográficas, como en broa, en su homónimo de «especie de galleta o bizcocho de maíz», que apareció por primer vez lematizada en la decimotercia edición del DRAE (1899) con la diferencia específica de que «se hace mucho en Filipinas» (especificación que a posteriori se suprimió), en donde Román se lamentaba irónicamente: «iPobres filipinos! Solo ahora que se han emancipado de España ha venido la Academia a admitirles este y otros vocablos» (1901-1908).

Ya había dado cuenta de budín y la petición de Román acerca de que se resolviera la forma en cómo adaptar el anglicismo, sin dar la cita completa, que la dejo en este apartado en donde se da cuenta de la ironía y la vehemencia que se podía tener con la institución hasta en sus grandes seguidores: «Resuelva la cuestión la Academia, pero no con la lentitud que acostumbra, sino con la buena voluntad de las personas laboriosas» (1901-1908).

\subsubsection{Pedir que la RAE enmiende la definición o parte de la definición}

Otra actitud usual dentro de la primera lexicografía hispanoamericana era la de proponer enmiendas al Diccionario académico, sobre todo de palabras relacionadas con Hispanoamérica. Tal es el caso de chancaca, entendida como la panela, pero de miel de remolacha azucarera en Chile y en la mayoría de los países hispanoamericanos con climas templados, de la que Román señala: «Azúcar mascabado en panes prismáticos, dice el último Dicc. El complemento «en panes prismáticos» debe suprimirse en la definición, porque no pertenece a la esencia de lo definido, sino que es solamente una de las muchas formas en que puede presentarse la chancaca» (1908-1911), forma que se modificó en otra a la actualidad: «Tableta rectangular hecha con la miel que se obtiene de la caña de azúcar» (DLE), lo que refleja, en todo caso, la forma que se ha impuesto de la chancaca misma. Como sea, Lenz describió las formas usuales en que se vendía a principios del siglo xx en Chile: «generalmente en líos de totora de forma más o menos cilíndrica, llamados mazos o lulos de chancaca» (1979 [1904-1910]).

\subsection{Aspectos de normatividad y corrección}

\subsubsection{Incorrecciones para Román}

Una de las funciones de un diccionario mixto como el de Román era, fuera de dar cuenta de las palabras características de Chile o de Hispanoamérica, ser un diccionario normativo, de consultas, de dudas. Por lo mismo, las palabras relacionadas con el campo semántico de los alimentos también tendrán este tratamiento. Por lo general, son cuestiones de variantes, algunas consideradas incorrectas por el sacerdote.

Es el caso del artículo bayonesa, cuyo lema es la variación de mayonesa: «No hay más bayonesa en castellano que la terminación $\mathrm{f}$. De bayonés, natural de Bayona o perteneciente a esta ciudad» (1901-1908). 
Soledad Chávez Fajardo. Lo que pueden decir de la comida chilena los diccionarios fundacionales. Primera parte

En chuchoca, comenta: «El pueblo pronuncia aquí chichoca y en el Ecuador chuchuca; en el Perú y en la Argentina, chuchoca. En esta forma debe pues entrar en el Dicc.» (1908-1911). En este caso, el sacerdote aboga por una de las variantes que es la más extendida.

En otros casos, la actitud purista relacionada con lo normado por la RAE hace que Román caiga en correcciones extremas, como en conserva, entendida, en este caso, como el relleno de las empanadas de fruta o de algunos hojaldres, uso que considera incorrecto, porque: «La conserva castellana es 'fruta hervida en agua con almíbar o miel, hasta el punto necesario para que se conserve [...]» (1901-1908), por lo que Román no aboga por la polisemia, algo usual hacia la fecha (no hay que olvidar la postura oficial de Bello censurando la polisemia en español, por ejemplo). Respecto a una última normatividad que Román enuncia: «Según esto no debemos decir duraznos, uvas, frutas en conserva (a lo sumo sería en conservación), sino Conservas de duraznos, uvas, etc., o Duraznos... conservados» (1901-1908), creo que el purismo es tanto, que al no aparecer la locución lematizada en el DRAE (apareció por primera vez en la vigésima edición, en 1984) Román descartó el uso, siendo que este («en conserva»), de seguir al CORDE, ya estaría textualizado entre los siglos XV y XVI.

\subsubsection{Cuestiones de adaptación de palabras de procedencia no hispánica}

De la mano con el tema de las adaptaciones de los extranjerismos que hace la RAE, se encuentra información respecto a las reflexiones del lexicógrafo mismo respecto a cómo adaptar determinada palabra, como las dudas vistas en cavial o caviar, la petición de hispanizar cognac en coñac o mantener la grafía original en curaçao.

En catrintre, que es el queso hecho con leche descremada, tomada por Román y por Lenz como un mapuchismo, así también en una parte de la tradición académica, hasta la supresión de la voz en la vigésima segunda edición de 2001. Román reflexionaba críticamente en torno a la adaptación del grupo tr mapudungun: «Y aquí protestamos de una vez por todas contra los araucanistas, que no acertaron a escribir el sonido araucano trra, trre, trri, trro, trru, sino que lo conviertieron en tha, the, thi, tho, thu, como si la h tuviera en castellano algún sonido» (1901-1908).

En el caso de consommé, por más que Román despotricara contra el galicismo y abogara por la forma consumado: «Hace más de veinte años que el Dicc. Lo tiene admitido en la forma castellana consumado» (1901-1908), terminó por imponerse la forma francesa simplificada, consomé, pero de manera lenta: marcada como incorrecta en las ediciones manuales de la RAE (1950), para pasar a ser remisión (decimonovena edición de 1970) y terminar como la variante que se impuso en la vigésima edición de 1992 y consumado se suprimió con esta acepción en esa misma edición.

Respecto a los alcoholes, de los que ya he hablado, las reflexiones en torno a su adaptación abundan: en el ya mencionado champaña vela Román por su hispanización: «inclúyase cuanto antes y así, a la española, champaña, pues muchos no saben cómo escribirlo y pronunciarlo. De las tres formas que suelen usarse champagne, champañ y champán, no es posible aceptar ninguna: hay que trajearlo necesariamente a la castellana» (1908-1911). 


\section{Soledad Chávez Fajardo. Lo que pueden decir de la comida chilena los diccionarios fundacionales. Primera parte}

En chinchibí, que es la cerveza de jengibre, Hartzenbusch en el Prólogo que escribió para el Diccionario de galicismos de Baralt, hipotetizando respecto a posibles y descabelladas adaptaciones, ejemplificaba: «Otro llamará yinyibia a la cerveza de jengibre» (1918 [1855]: XIII), a lo que Román replicaba: «pero no se conoce todavía al pueblo tan afeminado que use de una voz como esa» (1908-1913). Como sea, se generalizó la adaptación de chinchibí y apareció lematizada por primera vez en Gagini (1892), quien, dentro de mi rastreo en diccionarios es quien primero hace la mención y da cuentadle uso en «Chile y Costa rica». En el NTLLE: Zerolo, Alemany y Rodríguez Navas. Dentro de la tradición lexicográfica, solo en el Diccionario de Americanismos, para El Salvador, Nicaragua y Costa Rica.

\subsubsection{Cuestiones de normatividad}

Aspectos relacionados con el seseo americano en un diccionario de tono normativo como el de Román abundarán. No se escapa el campo de los alimentos. Es el caso del artículo lexicográfico cidra, en donde se da cuenta de los homófonos (en boca de un seseante) en donde cidra es el «Fruto del cidro» y, a continuación, en el mismo artículo: sidra «bebida alcohólica, de color ambarino, que se obtiene por la fermentación del zumo exprimido de las manzanas» (1901-1908). Para que quede claro que son dos signos absolutamente distintos.

En el ya mencionado chacoli, por ejemplo, se hace referencia a ciertas variantes de las que hay que prescindir: «La gente más ignorante suele pronunciar chocolá (1908-1913).

\subsection{Dar cuenta del referente: «en Chile existe este alimento»}

Una de las cuestiones más interesantes en esta investigación tiene que ver con detectar la enciclopedia alimenticia y gastronómica que está escondida dentro de un diccionario, sobre todo en esta lexicografía fundacional hispanoamericana. Me escaparía de los espacios estrictamente lexicológicos si explico contextualmente lo que ha implicado en la historia de Chile la migración campo ciudad desde la primera mitad del siglo xx, como primer hito para una baja de frecuencia de producción y consumo y de conocimiento de una serie de referentes que tienen relación con el campo de la alimentación. Como segundo hito, a partir de las lógicas y dinámicas neoliberales a partir de la dictadura de Pinochet, las que han instalado grandes transnacionales en territorio chileno, muchos usos, alimentos y costumbres han ido desapareciendo en pos de una alimentación más uniforme (la llamaría «la cultura de la comida procesada y citadina»). Es verdad que el concepto de slow food de los últimos años ha ido tomando un espacio clave en el rescate de muchísimas comidas y alimentos, pero aún queda mucho por hacer en Chile, por lo que creo que este apartado será un verdadero aporte cuando se finalice la investigación. 


\section{Soledad Chávez Fajardo. Lo que pueden decir de la comida chilena los diccionarios fundacionales. Primera parte}

\subsubsection{Alimentos dulces}

\subsubsection{Golosinas}

Un campo en peligro dentro de la gastronomía chilena es el de la confitería. Un grupo de dulces prácticamente han desaparecido de circulación (cfr. Ruperto de Nola ${ }^{3}$ 2015) o de usos y costumbres, como en el caso de bolada: «Manjares apetitosos que se llevan de afuera a personas encerradas, como colegiales, asilados, presos. En sentido fig., cualquier cosa buena que estaba oculta y llega a descubrirse. En ambas aceps. Es muy usado en Chile» (1901-1908). Quedará, como trabajo pendiente, cotejar la vigencia, textualizaciones y diatopía de bolada.

\subsubsection{Postres}

Anteriormente ya se había hecho referencia a la particularidad del alfajor, que Román describía para el caso de Chile como: «alfajor chileno, que es un dulce pequeño, formado de dos piezas de masa más o menos fina y adheridas la una a la otra con manjar blanco o melcocha» (1901-1908). También ya había mencionado la casi extinta cajetilla «Merengue vaciado y cocido en una especie de cajita de papel. Así se llama en Chile» (1901-1911), de la que Ruperto de Nola refiere, además, la complejidad en su preparación (2015). O la cocada y la extensión de su uso, a tal punto que ha terminado por ser, además, un americanismo etimológico, al dejar de ser marcada diatópicamente y popular en España.

En el caso de la referencia que hace Román al belado de bocado, el que cuenta con una preparación especial, que mezcla huevos, vainilla y azúcar, tiene que ver, más que nada, con aspectos de normatividad: «Adviértase que el sorbete español no es lo que aquí llamamos con ese nombre, sino que corresponde a nuestros helados» (1901-1908), en donde se muestra que en Chile tempranamente la distinción entre belado y sorbete se simplificó.

Un ejemplo prácticamente transversal en gran parte de Hispanoamérica es el postre llamado chimbo o buevo chimbo, con diversas variantes en su preparación. Aquí la chilena, en pluma de Román:

Chimbo, m. y ú. t. c. adj. Precedido del s. huevo. Dulce de yemas de huevo cocido en baño de maría y removido en almíbar. Por fuera lleva sobrepuestas pepitas de almendra, con lo cual en algo se parece a la tuna o higo chumbo, y quizás a este debe su nombre. También puede derivarse del quichua chimpu, arreboles o cerco del sol, por el color de este dulce. «En Maracaibo y Guatemala, como también en Bogotá, huevos chimbos es un dulce de yemas de huevo». (Cuervo). Lo mismo en el Perú. (Zerolo). El nombre castellano es yema: «dulce seco. Compuesto de azúcar y yema de huevo de gallina». Si tiene figura de capucha, se llama capuchina, f. (1908-1911)

He citado el artículo lexicográfico completo, el que es un buen ejemplo de lo que hacía Román en su Diccionario: la microestructura estaba compuesta por una definición en primer lugar. Suele agregar

3 Pseudónimo del crítico gastronómico Augusto Merino. 


\section{Soledad Chávez Fajardo. Lo que pueden decir de la comida chilena los diccionarios fundacionales. Primera parte}

información respecto a la etimología (en este caso, una etimología dudosa, lo mismo en Lenz) y, a posteriori, Román presenta autoridades las que, en este caso, extienden la diatopía del postre a otras zonas americanas. Suele, de haber sinonimia con el español ejemplar, presentar la forma (en este caso yema), la que considerará la correcta. Luego, por algunas características del referente, también da cuenta de la familia semántica del postre, con capuchina, que es el mismo postre pero con una forma distinta.

\subsubsection{Alimentos semilíquidos de consistencia espesa}

Usuales como alimentos semilíquidos que se toman como desayuno, sobre todo en zonas rurales, como el chercan «mazamorra de harina tostada con agua (o leche) caliente y azúcan», define Lenz (1979 [19041910]) o cocho, el que Román explica como una diatopía de chercan: «En algunas partes de Chile llaman así el ulpo [mazamorra de harina tostada con agua fría] caliente». Queda pendiente seguir trabajando en la vigencia y el uso de estas bebidas en Chile, así como precisar matices y variedades, pues he detectado que era este un desayuno absolutamente generalizado en el Chile rural antes de las grandes migraciones campo-ciudad y que sigue en vigencia en zonas rurales en la actualidad.

\subsubsection{Azúcar}

\subsubsection{Derivados a partir de la remolacha}

Como en el caso del ya mencionado chancaca, que Lenz confirma que la que consumía en Chile venía de Perú (¿O el tipo de preparación venía del uso peruano? Otro pendiente por investigar): «los vendedores en las calles de Santiago gritan ¡chancaca de Paita fina!» (1979 [1904-1910]). Sin embargo, chancaca será común en un gran número de países (cfr. DA y Fichero).

\subsubsection{Bebidas alcohólicas}

De las bebidas alcohólicas usuales en Chile, ya se hizo referencia al chacolí chileno: «vino hecho con la uva común del país, llamada de viña» (Román 1908-1911). También Román da cuenta de la diatopía del referente: «En algunas provincias lo confunden con la chicha, porque fermenta sin el orujo; en otras lo llaman vino nuevo; y de Maule al Sur, pitarrilla» (1908-1911).

Un actitud destacable, que refleja la tensión entre la norma monocéntrica y la variación, entre la relevancia de lo particular y la necesidad de darlo a conocer es el artículo lexicográfico chicha, que Román define de esta expresiva forma:

Chicha, f. ¡Quién les hiciera probar a los SS. Académicos la chicha de uvas, especialmente la de Aconcagua, para que la incluyeran en la definición! Véase la que han dado: «bebida alcohólica que resulta de la fermentación del maíz en agua azucarada y que se usa en América» (19081911). 


\section{Soledad Chávez Fajardo. Lo que pueden decir de la comida chilena los diccionarios fundacionales. Primera parte}

Este artículo lexicográfico afectivo (usual con algunos referentes del campo semántico de los alimentos), también es una manera de que en el DRAE extienda la diferencia específica en su artículo, algo que se terminó por hacer en la decimoctava edición de 1956, en una acepción especial para Chile, vigente hasta el día de hoy.

En el caso de chuflay, bebida que varía en el tipo de alcohol utilizado (puede ser pisco, aguardiente o cerveza) mezclada con limonada (actualmente gaseosa) o rodajas de limón por partes similares. Son Román y Lenz los primeros autores que la lematizan en un diccionario y, por razones que habrá que estudiar bien, ha permanecido en la lexicografía académica solo con la marca diatópica de Bolivia.

Para chupilca, bebida chilena, Lenz presenta variantes, una con harina tostada mezclada aguardiente de uva, chacolí o chicha (sea esta de uva, manzana u otra fruta) y otra variedad de chupilca de aguardiente con caldo de Sandía, harina tostada y aguardiente (cfr. 1979 [1904-1910]). La palabra fue lematizada por la tradición académica en la vigésimosegunda edición del DRAE, en 2001. En el caso de chupilca, se tiene, además, un cruce entre bebidas alcohólicas y alimentos semilíquidos de consistencia espesa, porque también forma parte de dicho campo y solía ser uno de los desayunos más consumidos en el campo chileno.

Respecto a la adaptación del kümmel (también conocida como gilka) a cominillo, tiene su primera referencia en Una excursión a los indios ranqueles, de Lucio V. Mansilla (1870, cfr Fichero) para Argentina. Por primera vez en un diccionario en Román: «Vulgarmente se llama también cominillo en Chile el licor llamado en alemán gilka» (1901-1908), le sigue Malaret, para Argentina, solamente (cfr. Fichero).

\subsubsection{Carnes}

Respecto a las carnes, Román hacía referencias a locuciones en desuso, como carne de Castilla: «llaman nuestro mayores la de cordero, sin duda porque la oveja fue introducida aquí por los que venían de Castilla, o a lo menos hablaban su idioma» (1901-1908), de la que no hay mayores registros.

\subsubsection{Casquería}

Respecto de la casquería, los intestinos de cordero y vaca que suelen cocinarse y asarse, han tomado en Chile la variante derivada del quechua chunchul, siempre en plural chunchules. Del mismo étimo derivaron: «En la Argentina dicen chinchulín, nes, y en Colombia chunchullos» (Román, 1908-1911), entre otros. Román, en su línea purista, suele, en estos casos, presentar la variedad castiza, entendida como la correcta: «En castellano tenemos revoltillo, 2. ${ }^{a}$ acep.: trenza o conjunto de tripas de carnero u otra res, que se forma revolviéndolas» (1908-1911).

$\mathrm{El}$ actual contre (la variante que se generalizó, también Lenz menciona un conti), que es la molleja de las aves, Román reconocía: «costará mucho sea desterrada por la molleja» (1901-1908), lo que da cuenta, algunas veces, del peso que tienen algunos indigenismos, absolutamente asentados en 


\section{Soledad Chávez Fajardo. Lo que pueden decir de la comida chilena los diccionarios fundacionales. Primera parte}

la comunidad hispanoamericana. Contri y contre fueron lematizados por primera vez dentro de la tradición académica en la decimonovena edición de 1970. Terminó por imponerse, dentro de la tradición académica, la variante contre en la última edición del DEL.

\subsubsection{Tipos de carnes}

El caso de charqui, la carne seca en la forma característica andina, palabra común en toda América del Sur y de gran consumo desde tiempos coloniales, Román reconocía: «este extendido americanismo, que no chilenismo, puesto que se usa en el Ecuador, Perú, Bolivia, Chile, Argentina, Uruguay y hasta en el Brasil» (1908-1911). Lenz hizo una reflexión clave respecto al charqui y la harina tostada en Chile: «Por su fácil preparación y valor alimenticio el charqui junto con la harina tostada todavía guarda su importancia para la vida del chileno en el campo, las minas y sobre todo en los viajes» (cfr. 1979 [1904-1910]). Su presencia en los diccionarios hispanoamericanos fundacionales es amplia (cfr. Lenz). Dentro de la tradición lexicográfica europea, Salvá, Gaspar y Roig, Domínguez, Zerolo y Toro y Gómez (cfr. NTLLE), todos con la variante en desuso charque. Román pide que, por su uso, sea incorporada la voz al DRAE: «Y por el uso tan general que tiene, creemos que está ya maduro para el Dicc.» (1908-1911). Charqui aparecerá lematizada en la décimocuarta edición de 1914 y remitida a tasajo.

\subsubsection{Cereales}

\subsubsection{Métodos de cocción de cereales}

Se tiene, por ejemplo la acción de culincar (o cullincar), forma de tostar granos característica de Wallmapu (la nación mapuche), que Lenz define como: «Tostar a medias el trigo, cebada, maíz o piñones (estos antes de cocerlos)» (cfr. 1979 [1904-1910]). El nombre del producto, el culincado «trigo u otro cereal medio tostado» (1901-1908), así como el proceso ya aparecían lematizados en el diccionario de Febrés en el xVIII (cfr. Lenz). No hay mayor difusión en la lexicografía española, salvo en Alemany y Rodríguez Navas (cfr. NTLLE).

\subsubsection{Comidas frías}

Un refrigerio usual de antaño y que cada vez se va restringiendo a ciertas preparaciones es el causeo: «Comida ligera que se toma, por lo general, a hora extraordinaria y por algún motivo particular, v.gr., por andar de paseo o de viaje. Ordinariamente se compone de alimentos fríos y secos, que pueden adquirirse y transportarse con facilidad» (1901-1908). Es un quechuismo: «La etimología de causeo es el quichua causay, o caucai, según lo escriben otros, y que significa la vida, la substistencia, las necesidades de la vida», (1901-1908) vinculado con la causa peruana. Deben haber estado tan conectados causeo y causa que Zorobabel Rodríguez y Lenz lematizaron causa para dicho artículo lexicográfico (cfr. 1979 [1904-1910]). Es otro de los indigenismos que Román encuentra tan asentados que ve difícil intentar, en su papel normativo, proponer una palabra 


\section{Soledad Chávez Fajardo. Lo que pueden decir de la comida chilena los diccionarios fundacionales. Primera parte}

equivalente: «La voz es de uso general en todo Chile y difícilmente se podrá reemplazar por otra; porque ni merienda, ni condumio, ni menos francachela, ni gaudeamus corresponden exactamente al concepto de causeo, que mejor, aunque de un modo muy genérico, se traduciría por refracción» (1901-1908).

\subsubsection{Condimentos}

La «Grasa derretida y preparada para condimentar los guisos» que define Román, que se compone básicamente de paprika o pimentón deshidratado picante, disueltos en esta grasa o en aceite actualmente, se conoce como la color y se suele acompañar como condimento de algunos guisos. Román explicaba: «Sin duda se le dio en Chile este nombre por el color que tiene (arrebolado o rojo amarillento) y porque además colora o da colorido a los mismos guisos» (1901-1908). La petición de Román respecto a que: «en castellano no puede tener equivalente, pues la color no se conoce en España, creemos que este chilenismo tiene derecho a la admisión» (1901-1908) no se ha concretado, salvo en el DA de la ASALE.

\subsubsection{Frutas}

\subsubsection{Frutas}

El mundo de la flora, con un basto campo por estudiar, sobre todo en pluma de botánicos, ha ayudado muchísimo a tipologizar un referente variado y, muchas veces, poco documentado dentro de la lexicografía hispanoamericana. Diccionarios como los de Román o Lenz serán fuentes relevantísimas de información al respecto, las más veces, alejada de un sesgo científico pero que conectará con el saber y conocimiento comunitario. Por ejemplo, para la familia prunus pérsica, llamada en Chile duraz̧no, se tiene el blanquillo: «un durazno parecido al de Zaragoza, pero de cáscara y corteza blancas» (1901-1908) o el durąno de bocado: «llámase aquí uno pequeño y pelado, que madura a principios del verano, y que en cuanto al hueso es abridero o abridor, porque el fruto se abre por medio y lo suelta con facilidad y limpio de carne, o en chileno, prisco» (1901-1908).

\subsubsection{Tipos de preparaciones con frutas}

Del tipo de preparación del charqui, como tasajos secados al sol, también pueden ser estos de alimentos que no sean carne, por eso, explicita Román: «En Chile significa también tajada, lonja o rebanada de algunas frutas, secada al sol y conservada para el invierno. Así hay charqui de membrillo, de tomate, de peras, de zapallo, de porotos» (1901-1908). Una costumbre, creo, que está cada vez más en retirada o, al menos, la nominación. 


\section{Soledad Chávez Fajardo. Lo que pueden decir de la comida chilena los diccionarios fundacionales. Primera parte}

\section{Conclusiones}

Es esta, como he dicho, una investigación en curso, la que tiene dos propósitos:

a) Por un lado, dar cuenta de lo que hay detrás de un artículo lexicográfico relacionado con el campo semántico de los alimentos en un caso característico de la lexicografía hispanoamericana fundacional, como es el del Diccionario de Román.

b) Por otro lado, presentar una propuesta de tipologización de esta información. Algo que en este primer estudio he decidido presentarla en su totalidad, aunque por motivos de espacio no pude desarrollar más que una parte.

Respecto al primer propósito, he detectado dos funciones en este tipo de artículos lexicográficos que tienen que ver con el normar y el mostrar.

a) El normar es una función transversal en este tipo de codificaciones. Tal como se ha visto, al analizar la información tratada por Román, lo que se detecta es un modelo altamente racionalista de estandarización, en donde abunda la intención perlocucionaria anteriormente referida, en donde la relevancia de lo que se está informando recae, las más veces, en las formas estandarizadas en que un alimento debe escribirse; adaptarse si es esta palabra un extranjerismo; qué palabra debe utilizarse, de haber sinonimia o posible sinonimia. En efecto, siguiendo a González Stephan (1995) y a Velleman (2004 y 2014), lo que se encuentra en estas codificaciones son escrituras disciplinarias, textos de misión civilizadora, como gramáticas, constituciones y manuales de urbanidad, por ejemplo, en donde el referente académico será constante.

b) El mostrar es una labor etnográfica clave para dar cuenta de la realidad hispanoamericana. En el caso de Román esto se da con la finalidad de que la información sea divulgada y, de cumplir con las condiciones lexicológicas necesarias que pide la RAE, sea incorporada en los repertorios académicos. Para Román, las más veces, esta función es indirecta, mas no en Lenz quien, alejado de estos propósitos racionalistas (dejo para otro estudio esta dicotomía Román/Lenz, la que por razones de espacio no pude desarrollar con mayor profundidad), ha llevado a cabo un trabajo más bien objetivo, que tiende a dar cuenta de un referente, sus nominaciones y sentidos, sus variedades, sin esperar una llegada a la RAE. El rescate de una serie de alimentos y usos alimenticios, creo, será uno de los grandes aportes del proyecto de investigación los que, en parte, se han visto en este estudio.

El propósito de muchos de estos artículos lexicográficos es dar noticia de que el DRAE lematizó cierta palabra o que la información que aparece de determinado alimento es insuficiente o que una palabra debe lematizarse por una serie de razones. Otras veces el mismo sacerdote reconocía la imposibilidad de imponer una voz castiza (considerada ejemplar) porque en el uso la palabra en cuestión ya está asentada. La tensión entre normar y mostrar; entre corregir y dar a conocer, aflora en varios de estos artículos lexicográficos y es esta muestra solo una punta del iceberg de una investigación de más largo aliento. 
Soledad Chávez Fajardo. Lo que pueden decir de la comida chilena los diccionarios fundacionales. Primera parte

\section{Bibliografía}

\section{Estudios:}

Chávez Fajardo, Soledad (2015) «Diccionarios precientíficos, productos lingüísticos otros». En: Álvarez López, Cristóbal José; Garrido Martín, Blanca y González Sanz, Marina: Jóvenes aportaciones a la investigación lingüistica. Sevilla, Alfar, 2015, pp. 187-198.

Chávez Fajardo, Soledad (2011) «Ideas lingüísticas de Lenz en los paratextos de su Diccionario etimológico de las voces chilenas derivadas de lenguas indígenas americanas». Lenguas modernas 38, pp. 83-106.

Geeraerts, Dirk (2003) «Cultural models of linguistics standarization». En: Dirven, René; Frank, Roslyn y Pütz, Martin (eds.) Cognitive models in language and thought. ideology, Metaphors and Meanings. Berlin, Mouton de Gruyter, pp. 25-68.

González Stephan, Beatriz (1995) «Las disciplinas escriturarias de la patria: constituciones, gramáticas y manuales». Estudios: Revista de investigaciones literarias 3(5), pp. 19-46.

Lara, Luis Fernando (1997) Teoría del Diccionario Monolingüe. México, El Colegio de México.

Nola, Ruperto de (2015) «Una historia de dulce y olvido». El Mercurio: http://www. economiaynegocios.cl/noticias/noticias.asp?id=181935 [última visita 18/11/21]

Pêcheux, Michel (2005 [1 $1^{\mathrm{a}}$ ed. 1975]) Semâtica e discurso. Uma crítica à afirmação do óbvio. Campinas, Editora da UNICAMP.

Rojas, Darío (2010) «Estandarización lingüística y pragmática del diccionario: forma y función de los "diccionarios de provincialismos" chilenos». Boletin de Filología de la Universidad de Chile XLV/1, pp. 209-233.

Velleman, Barry L. (2014) «Bello y las “escrituras disciplinarias”: diccionarios, gramáticas, ortografías». Boletin de Filología de la Universidad de Chile XLIX/1, pp. 179-192.

Velleman, Barry L. (2004) «Antiacademicismo lingüístico y comunidad hispánica: Sarmiento y Unamuno». En: Valle, José del y Gabriel-Stheeman, Luis (eds.) La batalla del idioma: la intelectualidad hispánica ante la lengua. Frankfurt/Madrid, Vervuert/Iberoamericana, pp. 35-65.

\section{Diccionarios:}

Baralt, Rafael María (1918 [1ª ed. 1855]) Diccionario de galicismos. Madrid, Imprenta gráfica excelsior.

Corominas, Joan (1980) Diccionario crítico etimológico castellano e hispánico. Madrid, Gredos. A-Ca.

Gagini, Carlos (1892) Diccionario de barbarismos y provincialismos de Costa Rica. San José de Costa Rica, Tip. Nacional.

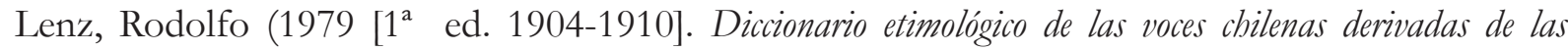
lenguas indigenas americanas. Edición dirigida por Mario Ferreccio. Santiago, Universidad de Chile, Seminario de Filología Hispánica.

SCRIPTA, Revista internacional de literatura i cultura medieval i moderna, núm. 18 / desembre 2021 / pp. 128-149 ISSN: 2340-4841· doi:10.7203/SCRIPTA.18.22768 
Soledad Chávez Fajardo. Lo que pueden decir de la comida chilena los diccionarios fundacionales. Primera parte

Román, Manuel Antonio (1908-1911) Diccionario de chilenismos y de otras voces y locuciones viciosas. Tomo II: CH, D, E, Fy suplemento á estas letras. Santiago, Imprenta de San José.

Román, Manuel Antonio (1901-1908) Diccionario de chilenismos y de otras voces y locuciones viciosas. Tomo I: A, B, C y suplemento á estas tres letras. Santiago, Imprenta de la Revista católica.

\section{Recursos en internet:}

Corpus Diacrónico del Español (CORDE): http://corpus.rae.es/cordenet.html

Corpus Diacrónico y Diatópico del Español de América (CORDIAM): http://www.cordiam.org/ Fichero General de la Lengua Española:

https://apps2.rae.es/fichero.html

Léxico Hispanoaméricano:

https://textred.spanport.lss.wisc.edu/index.html

Nuevo Tesoro Lexicográfico de la Lengua Española (NTLLE):

http://ntlle.rae.es/ntlle/SrvltGUILoginNtlle

Tesoro de los diccionarios históricos de la Lengua Española (TDHLE):

https://www.rae.es/tdhle/ 